\title{
Zero temperature dissipation and holography
}

\section{Pinaki Banerjee and B. Sathiapalan}

Institute of Mathematical Sciences, CIT Campus, Taramani, Chennai 600 113, India

E-mail: pinakib@imsc.res.in, bala@imsc.res.in

ABStract: We use holographic techniques to study the zero-temperature limit of dissipation for a Brownian particle moving in a strongly coupled CFT at finite temperature in various space-time dimensions. The dissipative term in the boundary theory for $\omega \rightarrow 0$, $T \rightarrow 0$ with $\omega / T$ held small and fixed, does not match the same at $T=0, \omega \rightarrow 0$. Thus the $T \rightarrow 0$ limit is not smooth for $\omega<T$. This phenomenon appears to be related to a confinement-deconfinement phase transition at $T=0$ in the field theory.

KEYWORDS: Gauge-gravity correspondence, Holography and quark-gluon plasmas, Black Holes, Thermal Field Theory

ArXiv EPRINT: 1512.06414 


\section{Contents}

1 Introduction 1

2 Dissipation at zero temperature $(T=0) \quad 3$

3 Dissipation near zero temperature $(T \rightarrow 0) \quad 5$

3.1 BTZ black hole background 5

$\begin{array}{lll}3.2 & \mathrm{AdS}_{5} \text { black hole background } & 6\end{array}$

4 Discussions 12

5 Conclusions 13

$\begin{array}{ll}\text { A Perturbative solution for string in BTZ } & 14\end{array}$

\section{Introduction}

The motion of an external heavy quark in a non-Abelian gauge theory has been studied in a number of papers (see [1-13] and references there in). One motivation for this are the experimental results that came out of the Relativistic Heavy Ion Collider (RHIC). The suggestion that the quark gluon plasma is "strongly coupled" with a very small value of $\frac{\eta}{s}$ came from experiments. The calculation of $\frac{\eta}{s}$ using AdS/CFT techniques [14-17] gave the small value of $\frac{1}{4 \pi}$ [18]. This gave impetus to holographic techniques for understanding the quark gluon plasma. On the dual gauge theory side exact calculations have been done for $\mathcal{N}=4$ super Yang-Mills theory - calculations that make heavy use of supersymmetry. One can hope that at finite temperature the deconfined QCD quark gluon plasma behaves qualitatively like strongly coupled $\mathcal{N}=4$ super Yang-Mills theory. The "running" effective coupling constant of QCD presumably is large in the field configurations that dominate the quark gluon plasma and therefore well approximated by a strongly coupled super YangMills theory. At finite temperature since supersymmetry is broken anyway and fermions and scalars effectively become massive, one can also presume that supersymmetry and the presence of adjoint fermions and scalars, does not invalidate the approximation. The success of the holographic calculation provides some indirect justification for all this.

Many calculations in super Yang-Mills have been done at zero temperature where supersymmetry is exact. In flat space, at all non zero temperatures, the theory is in the same Coulomb phase (unlike QCD) and therefore calculations done at zero temperature might be thought to still be of some relevance for the quark gluon plasma. On the dual gravity side this corresponds to pure $\mathrm{AdS}_{5}$. On the gravity side it is a little easier to explore the finite temperature regime [17]. This corresponds to non-extremal D3-branes. In fact the $\frac{\eta}{s}$ calculation involves calculating $\eta$ and $s$ separately at finite temperature. 
This paper studies the $T \rightarrow 0$ limit of the finite temperature calculations on the gravity side. This limit is subtle for reasons that will become clear later. The motivation for this study comes from an earlier paper [3] where the Langevin equation describing Brownian motion $^{1}$ of a stationary heavy quark in $1+1$ CFT at finite temperature was studied using the gravity dual, which is a BTZ black hole. The calculation was done using the holographic Schwinger-Keldysh method worked out in [2]. The calculation can be done exactly (unlike in $3+1$ dimensions). One of the interesting results is that there is a drag force (dissipating energy) on the fluctuating external quark even at zero temperature. This was identified as being due to radiation [5-11, 19-23]. This force term in the Langevin equation was of the form

$$
F(\omega)=-i \frac{\sqrt{\lambda}}{2 \pi} \omega^{3} x(\omega)
$$

If one calculates the integrated energy loss one finds $\left(x(t)=\int \frac{d \omega}{2 \pi} x(\omega) e^{-i \omega t}\right)$ :

$$
\begin{aligned}
\Delta E & =\int_{-\infty}^{\infty} F(t) \dot{x}(t) d t=\int \frac{d \omega}{2 \pi} F(w)(i \omega) x(-\omega)=\int \frac{d \omega}{2 \pi}(-i) \frac{\sqrt{\lambda}}{2 \pi} \omega^{3} x(\omega)(i \omega) x(-\omega) \\
& =\frac{\sqrt{\lambda}}{2 \pi} \int \frac{d \omega}{2 \pi} \omega^{4} x(\omega) x(-\omega)
\end{aligned}
$$

While the above calculation was done by taking the $T \rightarrow 0$ of a finite temperature calculation in BTZ, the same result is obtained for pure AdS in all dimensions, as shown in this paper. The energy radiated by an accelerating quark has been calculated using other techniques (also holographic) by Mikhailov [19] and the answer obtained is

$$
\Delta E=\frac{\sqrt{\lambda}}{2 \pi} \int d t a^{2}
$$

which on Fourier transforming gives exactly the same result. In fact the coefficient $\frac{\sqrt{\lambda}}{2 \pi}$ is essentially the bremsstrahlung function ${ }^{2} B(\lambda, N)\left(2 \pi B(\lambda, N)=\frac{\sqrt{\lambda}}{2 \pi}\right)$ identified in [28] as occurring in many other physical quantities (such as the cusp anomalous dimension introduced by Polyakov [29]).

It is thus interesting to check whether the same result is also obtained as one takes $T \rightarrow 0$ in $3+1$ dimensions. In fact the $T \rightarrow 0$ limit is a little tricky because of singularities. Taking $T \rightarrow 0$ in the finite $T$ theory can be done if one keeps $\frac{\omega}{T}$ small. Thus the limit where $\omega \rightarrow 0$ and $T \rightarrow 0$ is well defined and can be calculated perturbatively. (We shall see later that all the dimensionful quantities are measured in the unit of quark mass, $\mu$ ). The result for dissipation in this (DC) limit can be compared with that calculated for the $T=0$ result of pure AdS for $\omega \rightarrow 0$. The results do not agree. Thus we find that pure AdS $(T=0)$ results cannot be assumed to be close to small $T$ results as one would naively have assumed. This is not surprising given that there is a Hawking Page transition [30] at exactly zero temperature in the Poincare patch description of Schwarzschild-AdS. (In

\footnotetext{
${ }^{1}$ Brownian motion of a heavy quark in quark-gluon-plasma was first described using holographic techniques in $[1,2]$.

${ }^{2}$ See $[24-27]$ and references there in for more details about bremsstrahlung function in supersymmetric theories.
} 
global AdS where space is $S^{3}$, this happens at a finite temperature.) But this does raise questions of the relevance of the zero temperature calculations in $\mathcal{N}=4$ super Yang-Mills for comparison with data taken from experiments such as RHIC.

In this paper we study in a general way, the zero temperature limit of some theories in $3+1$ dimensions using holography. We show how one can do a perturbation in $T$ however this has to be done about a solution that is singular as $T \rightarrow 0$. This does not go smoothly to the $T=0$ result of pure AdS.

The rest of the paper is organized as follows. In section 2 we discuss dissipation at exactly zero temperature by studying dynamics of a long fundamental string in pure AdS space-time. We check whether the dissipation very close to zero temperature smoothly matches the same at absolute zero in section 3 by changing the background geometry for the moving string to AdS-black holes. In section 4 we try to interpret our results. The section 5 summarizes the main results obtained in this paper. The perturbative technique used in $\mathrm{AdS}_{5}-\mathrm{BH}$ case is also applied for studying string in BTZ background as a check of applicability of the method in appendix A.

\section{Dissipation at zero temperature $(T=0)$}

A Brownian particle dissipates energy at zero temperature only by radiating soft or massless modes (photons, gluons etc). The dual background where the string moves is a pure AdS space.

$$
d s^{2}=-\frac{r^{2}}{L^{2}} \mathrm{~d} t^{2}+\frac{L^{2}}{r^{2}} \mathrm{~d} r^{2}+\frac{r^{2}}{L^{2}} \mathrm{~d} \vec{x}^{2}
$$

$L$ is $\mathrm{AdS}$ radius and $\vec{x} \equiv\left(x^{1}, x^{2} \ldots x^{d-1}\right)$.

A stochastic string in this pure AdS background is exactly solvable in arbitrary dimensions. To compute the retarded Green's function and the dissipative term from that we need to study string dynamics in (2.1).

We shall be eventually working with linearized Nambu-Goto action. Therefore after choosing the static gauge, without loss of generality, we can pick up any one transverse direction $\left(x^{1} \equiv x\right.$, say) and fix others $\left(x^{2}, x^{3} \ldots x^{d-1}\right)$ to zero. Essentially we are looking at a three dimensional slice of $\mathrm{AdS}_{d+1}$. So, $\mathrm{X}(\sigma, \tau)$ is a map to $(\tau, r, x)$. The Nambu-Goto action for small fluctuation in space and in time reduces to

$$
\begin{aligned}
S & =-\frac{1}{2 \pi l_{s}^{2}} \int \mathrm{d} t \mathrm{~d} r \sqrt{1+\dot{x}^{2}+\frac{r^{4}}{L^{4}} x^{\prime 2}} \\
& \approx-\int \mathrm{d} t \mathrm{~d} r\left[1+\frac{m}{2} \dot{x}^{2}+\frac{1}{2} T_{0}(r) x^{\prime 2}\right]
\end{aligned}
$$

$m=\frac{1}{2 \pi l_{s}^{2}}$ and $T_{0}(r)=\frac{r^{4}}{2 \pi l_{s}^{2} L^{4}}$. Varying the action we get the EOM which in frequency space reads

$$
f_{\omega}^{\prime \prime}(r)+\frac{4}{r} f_{\omega}^{\prime}(r)+\frac{L^{4} \omega^{2}}{r^{4}} f_{\omega}(r)=0
$$


where $x(r, t)=\int \frac{\mathrm{d} \omega}{2 \pi} e^{-i \omega t} f_{\omega}(r) x_{0}(\omega)$ and $x_{0}(\omega)$ is the boundary value ${ }^{3}$ of $x(r, t)$ such that $f_{\omega}\left(r_{B}\right)=1$.

This is a linear second order ordinary differential equation with following two linearly independent solutions

$$
\begin{aligned}
f_{\omega}^{(1)}(r) & =\frac{e^{-i \frac{L^{2} \omega}{r}}\left(r+i L^{2} \omega\right)}{r} \\
f_{\omega}^{(2)}(r) & =\frac{e^{i \frac{L^{2} \omega}{r}}\left(r-i L^{2} \omega\right)}{r}
\end{aligned}
$$

- As we want to compute retarded Green's function we pick $f_{\omega}^{(2)}(r)$ which is ingoing ${ }^{4}$ at $r=0$.

- The boundary condition, $f_{\omega}(r) \rightarrow 1$ as $r \rightarrow r_{B}$, fixes the solution to be

$$
f_{\omega}(r)=\frac{r_{B}}{r} \frac{e^{+i \frac{L^{2} \omega}{r}}\left(r-i L^{2} \omega\right)}{e^{+i \frac{L^{2} \omega}{r_{B}}}\left(r_{B}-i L^{2} \omega\right)}
$$

We just use these modes in calculating the on-shell action and obtain the retarded Green's function [31, 32].

$$
\begin{aligned}
G_{R}^{0}(\omega) & \equiv \lim _{r \rightarrow r_{B}} T_{0}(r) f_{-\omega}(r) \partial_{r} f_{\omega}(r) \\
& =-\frac{r_{B}^{2} \omega^{2}}{2 \pi l_{s}^{2}} \frac{1}{\left(r_{B}-i L^{2} \omega\right)} \\
& =-i \frac{\mu^{2} \omega^{2}}{2 \pi \sqrt{\lambda}} \frac{1}{\left(\omega+i \frac{\mu}{\sqrt{\lambda}}\right)}
\end{aligned}
$$

where we have introduced a mass scale $\mu=\frac{r_{B}}{l_{s}^{2}}$ and a dimensionless parameter $\sqrt{\lambda}=\frac{L^{2}}{l_{s}^{2}}$. Notice that $\mu$ and $\lambda$ behave like Wilsonian cutoff scale and dimensionless coupling for the field theory respectively [3]. $\mu$ is essentially the energy stored in the string as it is stretched from the horizon to the boundary of the AdS. This is also interpreted as the mass of the external quark.

For $\mu \rightarrow \infty, G_{R}^{0}(\omega)=-\frac{\mu \omega^{2}}{2 \pi}$ which is divergent. We can renormalize the Green's function by absorbing the UV divergent piece to define the zero temperature mass of the quark, i.e, $M_{Q}^{0}=\frac{\mu}{2 \pi}$ and obtain the renormalized Green's function

$$
G_{R}(\omega) \equiv G_{R}^{0}(\omega)+\frac{\mu \omega^{2}}{2 \pi}=\frac{\mu \omega^{3}}{2 \pi} \frac{1}{\left(\omega+i \frac{\mu}{\sqrt{\lambda}}\right)}
$$

\footnotetext{
${ }^{3}$ Notice that $r=r_{B}$ is the boundary of the geometry. This is IR cutoff for the bulk and UV cutoff for the dual field theory. For large but finite value of $r_{B}$ the quark is very heavy but has a finite mass, and therefore, a detectable Brownian motion.

${ }^{4}$ Notice that $e^{-i \omega t} e^{i \frac{L^{2} \omega}{r}}=e^{-i \omega\left(t-\frac{L^{2}}{r}\right)}$. To keep the phase unchanged, for increasing $t, r$ must decrease. So the wave is ingoing.
} 
The renormalized Green's function is UV finite by construction. If we take $\mu \rightarrow \infty$ (or we can take $\omega$ very small)

$$
G_{R}(\omega) \rightarrow-i \frac{\sqrt{\lambda}}{2 \pi} \omega^{3}
$$

This is purely dissipative term which is independent of temperature.

\section{Dissipation near zero temperature $(T \rightarrow 0)$}

To describe a Brownian particle moving in a d-dimensional space-time at finite temperature holographically one needs to consider a fundamental string in $(d+1)$-dimensional dual geometry with a black hole. In this section also we work in the Poincare patch of $\mathrm{AdS}_{d+1}$-Black hole geometry.

We start with the $\mathrm{AdS}_{d+1}$-black brane metric [12]

$$
d s_{d+1}^{2}=L^{2}\left[-h(u) d t^{2}+\frac{\mathrm{d} u^{2}}{h(u)}+u^{2} \mathrm{~d} x^{2}\right]
$$

where $h(u)=u^{2}\left(1-\left(\frac{u_{h}}{u}\right)^{d}\right)$ with $u_{h}=\frac{4 \pi T}{d}$. $u$ has dimension of energy.

We choose $d=2$ and 4 for illustration. The aim is to check whether the dissipative terms match smoothly to that of the zero temperature case as we take $T \rightarrow 0$.

\subsection{BTZ black hole background}

A string in (2+1) dimension is exactly solvable even in presence of a (BTZ) black hole [1,3]. We work in (3.1) for $d=2$ but with $r$-coordinate where $r \equiv L^{2} u$ has dimension of length.

$$
d s^{2}=-\frac{r^{2}}{L^{2}}\left(1-\frac{\left(2 \pi T L^{2}\right)^{2}}{r^{2}}\right) \mathrm{d} t^{2}+\frac{L^{2}}{r^{2}} \frac{\mathrm{d} r^{2}}{\left(1-\frac{\left(2 \pi T L^{2}\right)^{2}}{r^{2}}\right)}+\frac{r^{2}}{L^{2}} \mathrm{~d} \vec{x}^{2}
$$

Using the holographic prescription for Minkowski space $[2,31]$ one obtains the exact retarded Green's function. Here are the key steps (see [3] for details).

Choosing the static gauge, we study small fluctuations of the string from the NambuGoto action

$$
S \approx-\int \mathrm{d} t \mathrm{~d} r\left[m+\frac{1}{2} T_{0}\left(\partial_{r} x\right)^{2}-\frac{m}{1-\left(\frac{2 \pi T L^{2}}{r}\right)^{2}}\left(\partial_{t} x\right)^{2}\right]
$$

where, $m \equiv \frac{1}{2 \pi l_{s}^{2}}$ and $T_{0}(r) \equiv \frac{1}{2 \pi l_{s}^{2}} \frac{r^{4}}{L^{4}}\left[1-\left(\frac{2 \pi T L^{2}}{r}\right)^{2}\right]$.

Now varying this action one obtains the EOM in frequency space

$$
f_{\omega}^{\prime \prime}(r)+\frac{2\left(2 r^{2}-4 \pi^{2} T^{2} L^{4}\right)}{r\left(r^{2}-4 \pi^{2} T^{2} L^{4}\right)} f_{\omega}^{\prime}(r)+\frac{L^{4} \omega^{2}}{\left(r^{2}-4 \pi^{2} T^{2} L^{4}\right)^{2}} f_{\omega}(r)=0
$$

which is exactly solvable and the solution is

$$
f_{\omega}(r)=C_{1} \frac{P_{1}^{\frac{i \omega}{2 \pi T}}\left(\frac{r}{2 \pi T L^{2}}\right)}{r}+C_{2} \frac{Q_{1}^{\frac{i \omega}{2 \pi T}}\left(\frac{r}{2 \pi T L^{2}}\right)}{r}
$$

where $P$ and $Q$ are associated Legendre functions. 
Now choosing ingoing boundary condition (to obtain retarded Green's function) at the horizon and fixing $f_{\omega}\left(r_{B}\right)=1$ one obtains the required solution

$$
f_{\omega}^{R}(r)=\frac{\left(1-\frac{r}{2 \pi T L^{2}}\right)^{-i \omega / 4 \pi T}}{\left(1-\frac{r_{B}}{2 \pi T L^{2}}\right)^{-i \omega / 4 \pi T}} \frac{\left(1+\frac{r}{2 \pi T L^{2}}\right)^{i \omega / 4 \pi T}}{\left(1+\frac{r_{B}}{2 \pi T L^{2}}\right)^{i \omega / 4 \pi T}} \frac{r_{B}}{r} \frac{\left(L^{2} \omega+i r\right)}{\left(L^{2} \omega+i r_{B}\right)}
$$

Now from the on-shell action we can read off the Green's function

$$
G_{R}^{0} \equiv \lim _{r \rightarrow r_{B}} T_{0}(r) f_{-\omega}^{R}(r) \partial_{r} f_{\omega}^{R}(r)=-\mu \omega \frac{\left(i \sqrt{\lambda} 4 \pi^{2} T^{2}+\mu \omega\right)}{2 \pi(\mu-i \sqrt{\lambda} \omega)}
$$

where we have used previously defined mass scale $\mu$ and the dimensionless parameter $\lambda$. For $\mu \rightarrow \infty, G_{R}^{0}(\omega)=-\frac{\mu \omega^{2}}{2 \pi}$ which is again divergent. We can renormalize the Green's function as before by absorbing the UV divergent piece to define the zero temperature mass of the quark, i.e, $M_{Q}^{0}=\frac{\mu}{2 \pi}$ and obtain the renormalized Green's function

$$
G_{R}(\omega) \equiv G_{R}^{0}(\omega)+\frac{\mu \omega^{2}}{2 \pi}=\frac{\mu \omega}{2 \pi} \frac{\left(\omega^{2}+4 \pi^{2} T^{2}\right)}{\left(\omega+i \frac{\mu}{\sqrt{\lambda}}\right)}
$$

Near zero temperature

$$
\left.G_{R}(\omega)\right|_{T \rightarrow 0}=\frac{\mu \omega^{3}}{2 \pi} \frac{1}{\left(\omega+i \frac{\mu}{\sqrt{\lambda}}\right)}
$$

This is identical to the retarded Green's function for zero temperature system (2.8). Evidently for small frequency

$$
G_{R}(\omega) \approx-i \frac{\sqrt{\lambda}}{2 \pi} \omega^{3}
$$

From this calculation black hole background seems to match smoothly pure AdS space as one takes $T \rightarrow 0$. The obvious question comes to one's mind is whether this coefficient of zero temperature dissipation is universal and independent of the dimensionality of space time. Actually we will see in the next section that this is not really the case in general. Reason being the Poincare patch of BTZ black hole is, strictly speaking, $\mathrm{AdS}_{3}$ at finite temperature for all practical purposes. That's why it smoothly matches the pure AdS result as one takes $T \rightarrow 0$. But $\operatorname{AdS}_{d+1}-\mathrm{BH}$ with $d>2$ is a "genuine" black hole background and therefore the limit might not be smooth.

\section{$3.2 \quad \mathrm{AdS}_{5}$ black hole background}

Now let us check if the dissipation coefficients for higher dimensional black holes in AdS space near zero temperature match the exact zero temperature coefficient. But those are not exactly solvable. As an example we will demonstrate it for $\mathrm{AdS}_{5}$ black hole [2].

We can write down the metric in $r$-coordinate, as before, fixing $d=4$ in (3.1)

$$
d s^{2}=-\frac{r^{2}}{L^{2}}\left(1-\frac{\left(\pi T L^{2}\right)^{4}}{r^{4}}\right) \mathrm{d} t^{2}+\frac{L^{2}}{r^{2}} \frac{\mathrm{d} r^{2}}{\left(1-\frac{\left(\pi T L^{2}\right)^{4}}{r^{4}}\right)}+\frac{r^{2}}{L^{2}} \mathrm{~d} \vec{x}^{2}
$$

with $\vec{x} \equiv\left(x^{1}, x^{2}, x^{3}\right)$. 
The $\mathrm{EOM}^{5}$ for the string

$$
F_{\omega}^{\prime \prime}(r)+\frac{4 r^{3}}{\left(r^{4}-\pi^{4} T^{4} L^{8}\right)} F_{\omega}^{\prime}(r)+\frac{\omega^{2} L^{4} r^{4}}{\left(r^{4}-\pi^{4} T^{4} L^{8}\right)^{2}} F_{\omega}(r)=0
$$

can not be solved exactly. As it's an ordinary second order linear differential equation it has two linearly independent solutions.

Near the horizon

$$
F_{\omega}(r) \sim\left(1-\frac{\pi^{4} T^{4} L^{8}}{r^{4}}\right)^{ \pm i \frac{\Omega}{4}}
$$

Near the boundary

$$
F_{\omega}(r)=\left(1+\frac{\Omega^{2}}{2 r^{2}}+\ldots\right)+\frac{\chi(\Omega)}{r^{3}}\left(1-\frac{\Omega^{2}}{10 r^{2}}+\ldots\right)
$$

contains a non-normalizable and a normalizable mode. Here $\Omega \equiv \frac{\omega}{\pi T}$.

But still one can solve it in perturbation expansion ${ }^{6}$ in small frequency

$$
F_{\omega}(r)=\left(1-\frac{\pi^{4} T^{4} L^{8}}{r^{4}}\right)^{-i \frac{\Omega}{4}}\left(1-i \Omega f_{1}(r)-\Omega^{2} f_{2}(r)+i \Omega^{3} f_{3}(r)+\ldots\right)
$$

Putting this ansatz into (3.10) we get hierarchy of differential equations. Solving them order by order in $\Omega \equiv \frac{\omega}{\pi T}$ in principle one can obtain the unknown functions, $f_{i}(r)$ where $i=1,2,3, \ldots$.

Few useful remarks on the perturbative solution before we actually obtain it.

1. This type of perturbative solution has been calculated in [2] by Son and Teaney. As the authors were mostly interested in finite temperature phenomena they computed the Green's function up to $\omega^{2}$ term. In this article we show that their solution can be used even for $T \rightarrow 0$ and also we compute the Green's function up to $\omega^{3}$ term which indicates the zero temperature dissipation.

2. The solution we obtain is a perturbation in $\Omega$ and $T$. But finally we are interested in $T \rightarrow 0$ limit. Clearly this limit is pathological for any finite $\omega$. Only way we can make sense of the solution is by taking both

$$
\omega, T \rightarrow 0 \text { with } \Omega \text { held fixed (and small). }
$$

The temperature independent term that we are interested in is the coefficient of $\Omega^{3} T^{3}$ in this solution.

It is important to note, all dimensionful quantifies in this theory are measured in terms of the cutoff scale $\mu\left(=\frac{r_{B}}{l_{s}^{2}}\right)$ defined before which is also interpreted as the

\footnotetext{
${ }^{5}$ Notice that for this case $m \equiv \frac{1}{2 \pi l_{s}^{2}}$ and $T_{0}(r) \equiv \frac{1}{2 \pi l_{s}^{2}} \frac{r^{4}}{L^{4}}\left[1-\left(\frac{\pi T L^{2}}{r}\right)^{4}\right]$. But eventually we want to calculate Green's function for $T \rightarrow 0$. Therefore for both BTZ and AdS-BH background we can practically use $T_{0}(r) \equiv \frac{1}{2 \pi l_{s}^{2}} \frac{r^{4}}{L^{4}}$.

${ }^{6}$ The -ve sign in the exponent is chosen in (3.12) which is ingoing at the horizon. Because we are interested to calculate the retarded Green's function.
} 
mass of the external quark. Therefore whenever we say $\omega, T \rightarrow 0$ with $\Omega$ held fixed we mean $\omega / \mu \rightarrow 0$ and $T / \mu \rightarrow 0$ such a way that $\omega / T$ is fixed small number. E.g., say, $\omega / \mu=10^{-7}, T / \mu=10^{-6}$, thus $\omega / T=0.1$ which is smaller than 1 .

3. Actually, as we will see below, we don't need to obtain all the unknown functions, $f_{i}(r)$, explicitly by performing complicated integrals. Rather we need only the residues of those integrals at the horizon to fix the coefficient of the homogeneous solutions.

\section{Perturbative solution in $\mathrm{AdS}_{5}-\mathrm{BH}$}

Just for convenience we work with $z$ co-ordinate, where $z \equiv \frac{L^{2}}{r}$. Obtaining results in the $r$ variable is straightforward. As we have discussed the EOM (3.9) for the string in $\mathrm{AdS}_{5}-\mathrm{BH}$

$$
F_{\omega}^{\prime \prime}(z)+\frac{2\left(1+\pi^{4} T^{4} z^{4}\right)}{z\left(1-\pi^{4} T^{4} z^{4}\right)} F_{\omega}^{\prime}(z)+\frac{\omega^{2}}{\left(1-\pi^{4} T^{4} z^{4}\right)^{2}} F_{\omega}(z)=0
$$

is not exactly solvable and to obtain the solution that is ingoing at the horizon we need to use the following ansatz.

$$
\begin{aligned}
F_{\omega}^{R}(z) & =\left(1-\pi^{4} T^{4} z^{4}\right)^{-i \frac{\Omega}{4}} H(z) \\
\text { where } H(z) & =1-i \Omega h_{1}(z)-\Omega^{2} h_{2}(z)+i \Omega^{3} h_{3}(z)+\ldots
\end{aligned}
$$

with $\Omega \equiv \frac{\omega}{\pi T}$.

The differential equation $H(z)$ satisfies is given by

$H^{\prime \prime}(z)-\frac{2\left(1+\pi^{3} T^{3} z^{4}(\pi T-i \omega)\right)}{z\left(1-\pi^{4} T^{4} z^{4}\right)} H^{\prime}(z)+\frac{\omega\left(\omega+\pi^{2} T^{2} z^{2}(\omega+i \pi T)\left(\pi^{2} T^{2} z^{2}+1\right)\right)}{\left(\pi^{2} T^{2} z^{2}+1\right)\left(1-\pi^{4} T^{4} z^{4}\right)} H(z)=0$

Notice that by choosing the ansatz (3.14) we have taken care of the singular near horizon part of the full solution by the pre-factor $\left(1-\pi^{4} T^{4} z^{4}\right)^{-i \frac{\Omega}{4}}$. Our strategy would be to substitute the ansatz (3.14) into (3.15) and at each order in $\Omega$ we demand that the solution to (3.15) is regular at the horizon. In other words, the full solution to (3.13) at any order in $\Omega$ behaves like

$$
\left(1-\pi^{4} T^{4} z^{4}\right)^{-i \frac{\Omega}{4}} \times\left(\text { Regular function at } z=\frac{1}{\pi T}\right)
$$

Again, we are interested in calculating temperature independent dissipative term in the Green's function. Therefore on dimensional ground we need to look for the coefficient of $\omega^{3}$ term in the Green's function as the Green's function itself has mass dimension three. In other words, if one takes zero temperature limit of the Green's function only $\omega^{3}$ term survives. For that one needs to take $T \rightarrow 0$ limit of the solution. But clearly the solution is a perturbation series in $\Omega=\frac{\omega}{\pi T}$. Therefore the only way one can make sense of this solution near zero temperature is to take both $T \rightarrow 0$ and $\omega \rightarrow 0$ (compared to $\mu$ ) keeping the perturbation parameter, $\Omega$ fixed and small $(\Omega<1)$ such that the series converges. 


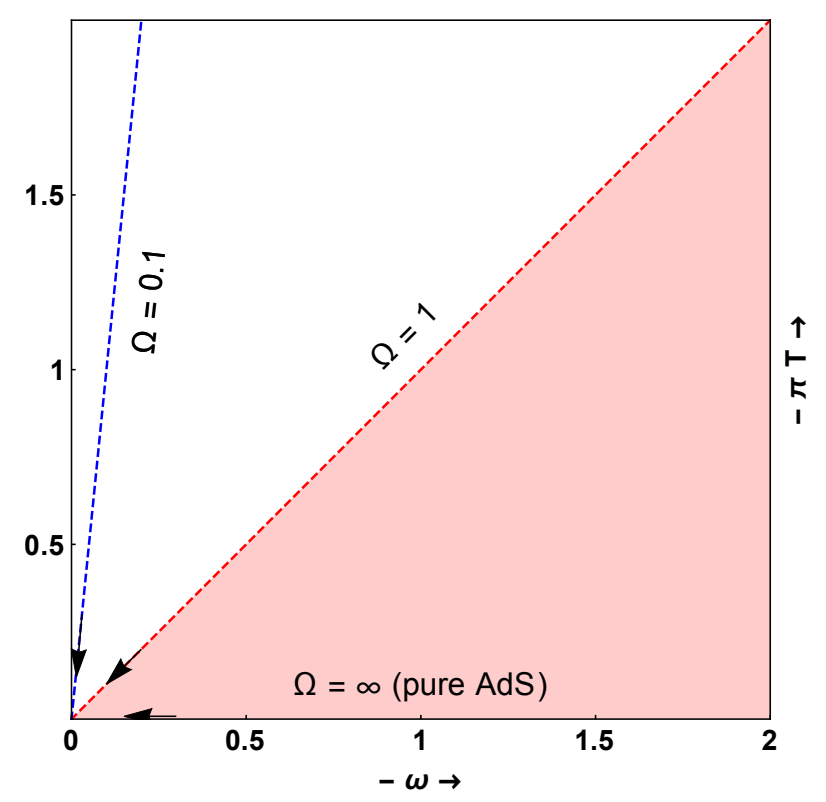

Figure 1. Different ways of taking $T=0, \omega=0$ limit.

Pictorially, there are many ways one can reach $(\omega=0, T=0)$ i.e, the origin on the $\omega-T$ plane (see figure 1). Our solution makes sense when $\omega / \pi T$ is a constant and smaller than one. The shaded region is outside the domain of validity of our solution. Thus all our analysis and results hold true for the straight lines in the upper half of the box.

Also notice that pure AdS $(T=0)$ is along the $x$-axis. Therefore eventually we will be comparing these two ways $(\Omega=$ small and $\Omega=\infty)$ of taking limits.

\section{Solution up to $\mathcal{O}(\Omega)$.}

$$
H(z)=1-i \Omega h_{1}(z)
$$

Substituting this ansatz into (3.13) we obtain the differential equation for $h_{1}(z)$

$$
h_{1}^{\prime \prime}(z)+\underbrace{\frac{2\left(1+\pi^{4} T^{4} z^{4}\right)}{z\left(1-\pi^{4} T^{4} z^{4}\right)}}_{p_{1}(z)} h_{1}^{\prime}(z)=\underbrace{\frac{\pi^{4} T^{4} z^{2}}{\left(1-\pi^{4} T^{4} z^{4}\right)}}_{q_{1}(z)}
$$

Lets cast this into a first order differential equation defining $y_{1}(z) \equiv h_{1}^{\prime}(z)$ and consequently $y_{1}^{\prime}(z) \equiv h_{1}^{\prime \prime}(z)$

$$
y_{1}^{\prime}(z)+p_{1}(z) y_{1}(z)=q_{1}(z)
$$

One can introduce integrating factor $I_{1}(z)=\exp \left(\int p_{1}(z) \mathrm{d} z\right)$ to obtain

$$
\begin{aligned}
y_{1}(z) & =\frac{c_{1}}{I_{1}(z)}+\frac{1}{I_{1}(z)} \int^{z} I_{1}(x) q_{1}(x) \mathrm{d} x \\
& =\underbrace{\frac{c_{1} z^{2}}{1-\pi^{4} T^{4} z^{4}}}_{y_{1}^{h}(z)}+\underbrace{\frac{\pi^{4} T^{4} z^{3}}{1-\pi^{4} T^{4} z^{4}}}_{y_{1}^{p}(z)}
\end{aligned}
$$


We will see that the homogeneous part of the solution $\left(y_{i}^{h}(z)\right)$ is identical in each order in $\Omega$ up to the undetermined coefficient $\left(c_{i}\right)$. This coefficient is fixed by demanding the regularity of $f_{i}$ at the horizon.

$$
\begin{aligned}
f_{1}(z) & =\int y_{1}^{h}(z) \mathrm{d} z+\int y_{1}^{p}(z) \mathrm{d} z \\
& \equiv h_{1}^{h}(z)+h_{1}^{p}(z)
\end{aligned}
$$

The requirement that $h_{1}(z)$ has to be regular sets the coefficient of $\log (1-\pi T z)$ to zero. One can explicitly calculate the integrals and from that expression sort out the required coefficient. For this case

$$
\begin{aligned}
h_{1}^{h}(z) & =\frac{c_{1}}{4 \pi^{3} T^{3}}\left\{-2 \tan ^{-1}(\pi T z)-\log (1-\pi T z)+\log (1+\pi T z)\right\} \\
h_{1}^{p}(z) & =-\frac{1}{4} \log \left(1-\pi^{4} T^{4} z^{4}\right) \\
& =-\frac{1}{4}\left\{\log (1-\pi T z)+\log (1+\pi T z)+\log \left(1+\pi^{2} T^{2} z^{2}\right)\right\}
\end{aligned}
$$

Clearly setting the coefficient of $\log (1-\pi T z)$ to zero we get

$$
c_{1}=-\pi^{3} T^{3}
$$

And the solution at this order becomes

$$
h_{1}(z)=\frac{1}{2} \tan ^{-1}(\pi T z)-\frac{1}{2} \log (1+\pi T z)+\frac{1}{4} \log \left(1+\pi^{2} T^{2} z^{2}\right)
$$

But there is another way in which we can fix the coefficient without doing the integrals. The only potential singular term in $h_{1}(z)$ at the horizon appears as $\log (1-\pi T z)$. This type of terms are originated from the terms of the form $\frac{1}{(1-\pi T z)}$ in $y_{1}(z)$. Therefore fixing the coefficient of $\log (1-\pi T z)$ in $f_{1}(z)$ to zero is equivalent to setting the residue of $y_{1}(z)$ at $z=\frac{1}{\pi T}$ to zero. This is much easier way when the integrals get complicated as we go in higher orders.

\section{Solution up to $\mathcal{O}\left(\Omega^{2}\right)$.}

$$
H(z)=1-i \Omega h_{1}(z)-\Omega^{2} h_{2}(z)
$$

Notice that $h_{1}(z)$ is already known from (3.24). Substituting this ansatz into (3.15) we get the differential equation for $h_{2}(z)$. Again one can cast that into a first order differential equation

$$
y_{2}^{\prime}(z)+p_{2}(z) y_{2}(z)=q_{2}(z)
$$

where $p_{2}(z)=p_{1}(z)$. Therefore integrating factor $I_{2}(z)=I_{1}(z)$.

$$
\begin{aligned}
y_{2}(z) & =\frac{c_{2}}{I_{2}(z)}+\frac{1}{I_{2}(z)} \int^{z} I_{2}(x) q_{2}(x) \mathrm{d} x \\
& =\underbrace{\frac{c_{2} z^{2}}{1-\pi^{4} T^{4} z^{4}}}_{y_{2}^{h}(z)}+\underbrace{\frac{1}{I_{2}(z)} \int^{z} I_{2}(x) q_{2}(x) \mathrm{d} x}_{y_{2}^{p}(z)}
\end{aligned}
$$


Now making the residue of $y_{2}(z)$ at $z=\frac{1}{\pi T}$ to vanish we can fix

$$
c_{2}=\pi^{3} T^{3}
$$

The solution at this order

$$
\begin{aligned}
h_{2}(z)= & \frac{1}{32}\left[4\left\{-4+\tan ^{-1}(\pi T z)-\log (1+\pi T z)\right\}\left\{\tan ^{-1}(\pi T z)-\log (1+\pi T z)\right\}\right. \\
& \left.-4\left\{2+\tan ^{-1}(\pi T z)-\log (1+\pi T z)\right\} \log \left(1+\pi^{2} T^{2} z^{2}\right)+\log \left(1+\pi^{2} T^{2} z^{2}\right)^{2}\right]
\end{aligned}
$$

Solution up to $\mathcal{O}\left(\Omega^{3}\right)$.

$$
H(z)=1-i \Omega h_{1}(z)-\Omega^{2} h_{2}(z)+i \Omega^{3} h_{3}(z)
$$

where $h_{1}, h_{2}$ are known from (3.24) and (3.28). The differential equation for $h_{3}(z)$ or rather $y_{3}(z) \equiv h_{3}^{\prime}(z)$

$$
y_{3}^{\prime}(z)+p_{3}(z) y_{2}(z)=q_{3}(z)
$$

where $p_{3}(z)=p_{1}(z)$. Therefore integrating factor $I_{3}(z)=I_{1}(z)$, as before.

$$
\begin{aligned}
y_{3}(z) & =\frac{c_{3}}{I_{3}(z)}+\frac{1}{I_{3}(z)} \int^{z} I_{3}(x) q_{3}(x) \mathrm{d} x \\
& =\underbrace{\frac{c_{3} z^{2}}{1-\pi^{4} T^{4} z^{4}}}_{y_{3}^{h}(z)}+\underbrace{\frac{1}{I_{3}(z)} \int^{z} I_{3}(x) q_{3}(x) \mathrm{d} x}_{y_{3}^{p}(z)}
\end{aligned}
$$

Now making the residue of $y_{3}(z)$ at $z=\frac{1}{\pi T}$ to vanish we obtain

$$
c_{3}=\left(\frac{\pi-\log 4}{4}\right) \pi^{3} T^{3}
$$

The functional form of $y_{3}(z)$ is complicated and therefore it's difficult to obtain an explicit expression for $h_{3}(z)$ unlike the lower order functions. But as we are interested in zero temperature limit of the Green's function, we only need to know the full solution for small $\Omega$

$$
\begin{aligned}
F_{\omega}^{R}(z)= & \left(1-\pi^{4} T^{4} z^{4}\right)^{-i \frac{\Omega}{4}}\left(1-i \Omega h_{1}(z)-\Omega^{2} h_{2}(z)+i \Omega^{3} h_{3}(z)\right) \\
\approx & \left(1+\frac{i}{4} \pi^{3} \omega T^{3} z^{4}\right)\left\{1-\frac{i \omega}{\pi T}\left(-\frac{1}{3} \pi^{3} T^{3} z^{3}\right)-\frac{\omega^{2}}{\pi^{2} T^{2}}\left(-\frac{1}{3} \pi^{2} T^{2} z^{2}\right)\right. \\
& \left.-\frac{i \omega^{3}}{\pi^{3} T^{3}}\left(\frac{\pi-\log 4}{12}\right) \pi^{3} z^{3} T^{3}\right\}
\end{aligned}
$$

Evidently in the zero temperature limit (with very very small frequency)

$$
\left.F_{\omega}^{R}(z)\right|_{T \rightarrow 0}=1+\frac{\omega^{2} z^{2}}{2}+i \frac{\omega^{3} z^{3}}{3}\left(\frac{\pi-\log 4}{4}\right)
$$

In $r$ co-ordinate

$$
\left.F_{\omega}^{R}(r)\right|_{T \rightarrow 0}=1+\frac{\omega^{2} L^{4}}{2 r^{2}}+i \frac{\omega^{3} L^{6}}{3 r^{3}}\left(\frac{\pi-\log 4}{4}\right)
$$


The retarded Green's function at $T \rightarrow 0$ (with $\omega \rightarrow 0$ and $\Omega=$ fixed) can be calculated using the solution (3.34)

$$
\begin{aligned}
G_{R}^{0} & \equiv \lim _{r \rightarrow r_{B}} T_{0}(r) F_{-\omega}^{R}(r) \partial_{r} F_{\omega}^{R}(r) \\
& =\lim _{r \rightarrow r_{B}} \frac{1}{2 \pi l_{s}^{2}} \frac{r^{4}}{L^{4}}\left(-\frac{\omega^{2} L^{4}}{r^{3}}-i \frac{\omega^{3} L^{6}}{4 r^{4}}(\pi-\log 4)\right) \\
& =-\frac{\mu \omega^{2}}{2 \pi}-i \frac{\sqrt{\lambda}}{2 \pi}\left(\frac{\pi-\log 4}{4}\right) \omega^{3}
\end{aligned}
$$

Therefore the renormalized Green's function

$$
G_{R}(\omega) \equiv G_{R}^{0}+\frac{\mu \omega^{2}}{2 \pi}=-i \frac{\sqrt{\lambda}}{2 \pi}\left(\frac{\pi-\log 4}{4}\right) \omega^{3}
$$

It is evident that this zero temperature dissipation term is not same as that of pure AdS case and actually off by a factor of $\frac{\pi-\log 4}{4}$.

\section{Discussions}

Whenever a charged particle accelerates or decelerates it radiates energy which is known as bremsstrahlung effect. In this paper we talk about dissipation at and near zero temperature. Naturally this zero temperature dissipation finds its origin in this bremsstrahlung phenomenon. One can notice that this dissipative force term $\left(\mathcal{F}_{\text {diss }}\right)$ goes as a cubic power in frequency

$$
\mathcal{F}_{\text {diss }}(\omega) \sim-i \sqrt{\lambda} \omega^{3} x(\omega)
$$

In real space this each $i \omega$ gives a time derivative and therefore the above force law reduces to

$$
\mathcal{F}_{\text {diss }}(t) \sim \sqrt{\lambda} \dddot{x}(t)=\sqrt{\lambda} \dot{a}
$$

$\dot{a}$ here quantifies the rate of change in acceleration and is often called jerk or jolt. This formula is very similar to that of "Abraham-Lorentz force" [33] in classical electrodynamics for a charged particle with charge $q$

$$
\mathcal{F}_{\text {rad }}(t)=\frac{2}{3} q^{2} \dot{a}
$$

This is the force that an accelerating charged particle feels in the recoil from the emission of radiation. Only the effective coupling is different for the holographic case. This "coupling" $(\sqrt{\lambda})$ is essentially the bremsstrahlung function $B(\lambda, N)$. The corrections in $\lambda$ and $N$ can also be computed for particular known theories (see [24-27]).

The main aim in this paper is to understand how this bremsstrahlung function behaves near zero temperature. We work in Poincare patch of AdS-black hole on the gravity side. We notice that for higher dimensional cases (we performed calculations in $\mathrm{AdS}_{5}-\mathrm{BH}$ ) value of this function ${ }^{7}$ near $T \rightarrow 0$ doesn't match that of at $T=0$. Strictly speaking, this result

\footnotetext{
${ }^{7}$ Of course we are working in leading order in large $N$ and large $\lambda$. To compute corrections one needs to work with some known supersymmetric UV theories (e.g., ABJM, $\mathcal{N}=4 \mathrm{SYM}$ ).
} 
is obtained in a particular regime of the parameter space namely $\omega / \mu \rightarrow 0$ and $T / \mu \rightarrow 0$ such a way that $\Omega<1$. Thus the domain of validity of our analysis is spanned by family of straight lines (see figure 1) which end at the origin and with slope greater than one (i.e, $\frac{\pi T}{\omega}>1$ ). It is important to note, $\omega \rightarrow 0$ limit for the solution to the pure AdS case is actually represented by the $x$-axis of figure 1 . Therefore effectively we are comparing two different ways ${ }^{8}$ of taking $T=0, \omega=0$ limit, "almost vertical lines" (AdS-BH) and "the horizontal line" (pure AdS). They don't match. Actually this result is not unexpected as there is a Hawking-Page phase transition at zero temperature in Poincare patch of black holes in AdS.

Therefore the result suggests that one should not use the $T=0$ theory (which always has $\Omega=\infty$ ) as an approximation to small temperature physics, when $\Omega$ is small i.e, $\omega<T$. Thus for example if $T=10^{-6}$ and $\omega=10^{-7}$ compared to $\mu$, we cannot use the $T=0$ theory. This is something that can be very crucial in the context of quarkgluon-plasma (QGP). QGP is always at finite temperature and therefore dissipation term more specifically the bremsstrahlung function $B(\lambda)$ is not continuously connected to the zero temperature background (pure AdS) result, at least for small frequencies $(\Omega<1)$. Therefore one must use the AdS-BH background to compute those quantifies even at very small temperature.

Unlike the higher dimensional case, we see that for a particle in $1+1$ dimensional field theory the bremsstrahlung functions match smoothly at $T=0$. The possible reason behind this phenomenon is hidden in the corresponding dual geometries namely $\mathrm{AdS}_{3}$ (for $T=0$ ) and BTZ (at $T \neq 0)$. A BTZ black hole is just an orbifold of $\mathrm{AdS}_{3}$ and therefore locally $\mathrm{AdS}_{3}$. One can only distinguish the former from the latter by studying global properties. BTZ in Poincare patch is no different than $\mathrm{AdS}_{3}$ at finite temperature (also called thermal $\mathrm{AdS}_{3}$ ) unlike the higher dimensional black holes in AdS which are "genuine" black hole backgrounds.

\section{Conclusions}

We summarize the main points here:

- We have studied Brownian motion in various space time dimensions with the help of holographic Green's function computation. In each case we obtain dissipation at zero temperature due to radiation from accelerated charged Brownian particle.

- As long as we are considering dynamics of the quark at zero temperature, that is the string in the dual gravity theory moves in a pure AdS spacetime, the coefficients of dissipation for arbitrary space time dimensions are identical. The value of the coefficient is $\frac{\sqrt{\lambda}}{2 \pi}$ and can be identified with $B(\lambda, N)[28]$ (actually $2 \pi B(\lambda, N)=\frac{\sqrt{\lambda}}{2 \pi}$ ) as occurring in many other physical quantities (such as the cusp anomalous dimension introduced by Polyakov [29]).

\footnotetext{
${ }^{8}$ It is worthwhile noting that the lines that end on the origin and are contained inside the shaded region are also valid ways of taking $T=0, \omega=0$ limit. But our analysis doesn't work for that region. Because in that region $\Omega>1$ and therefore our perturbative expansion (3.14) breaks down. Thus we cannot say much about high frequency domain with this analysis.
} 
- Even the coefficients match for string in $\mathrm{AdS}_{3}$ and in BTZ as we take $T \rightarrow 0$. This is because BTZ in Poincare patch is nothing more than a thermal $\mathrm{AdS}_{3}$.

- For higher dimensions the coefficients at $T=0$ and $T \rightarrow 0$ don't match for small frequencies $(\Omega<1)$. Here we are effectively comparing infinite $\Omega$ to finite and small $\Omega$ and they turn out to be different although both refer to the same region around $\omega=0, T=0$. Thus one should be careful in using pure AdS for calculating near zero temperature quantities (e.g., $B(\lambda)$ ) for very low (near zero) frequencies, i.e. $\Omega<1$. Even if the temperature is very very small (unless it's exactly zero) one should not use $T=0$ results as the $T=0$ and $T \rightarrow 0$ systems are described by completely different theories. We have shown this phenomenon via explicit computation by studying a string dynamics in $\mathrm{AdS}_{5}-\mathrm{BH}$ background. The corresponding coefficient comes out to be $\frac{\sqrt{\lambda}}{2 \pi} \frac{\pi-\log 4}{4}$. This phenomenon might have its origin in the Hawking-Page transition at $T=0$ in Poincare patch.

\section{A Perturbative solution for string in BTZ}

We already mentioned that EOM for a string in BTZ can be solved exactly and hence one can compute exact retarded Green's function for the Brownian particle. Actually we have done the same in 3.2. There we have seen that the zero temperature dissipation coefficient is $\frac{\sqrt{\lambda}}{2 \pi}$. On the other hand, the EOM of string in $\mathrm{AdS}_{5}-\mathrm{BH}$ is not exactly solvable and therefore we adopted a perturbative technique to compute the above mentioned coefficient. In 3.2 we got a different value for that coefficient. In this section we apply the same perturbative method for a string in BTZ and show that the same result for zero temperature dissipation is reproduced. This is just to show that the perturbative approach and the associated limits indeed work.

Our aim is to perturbatively solve (3.4) which in $z$-coordinate looks

$$
f_{\omega}^{\prime \prime}(z)+\frac{2}{z\left(1-4 \pi^{2} T^{2} z^{2}\right)} f_{\omega}^{\prime}(z)+\frac{\omega^{2}}{\left(1-4 \pi^{2} T^{2} z^{2}\right)^{2}} f_{\omega}(z)=0
$$

using the following ansatz

$$
f_{\omega}^{R}(z)=\left(1-4 \pi^{2} T^{2} z^{2}\right)^{-i \frac{\Omega}{2}}\left(1-i \Omega h_{1}(z)-\Omega^{2} h_{2}(z)+i \Omega^{3} h_{3}(z)+\ldots\right)
$$

where in this section $\Omega \equiv \frac{\omega}{2 \pi T}$ (notice the extra factor of two).

Again we will be working in the regime where

$$
\omega, T \rightarrow 0 \text { with } \Omega \text { held fixed (and small). }
$$

Next step is to solve for the unknown functions, $h_{1}(x), h_{2}(x), h_{3}(x)$ etc. recursively. We repeat the same procedure as in (3.2). 
Solution up to $\mathcal{O}(\Omega)$.

$$
f_{\omega}(z)=\left(1-4 \pi^{2} T^{2} z^{2}\right)^{-i \frac{\Omega}{2}}\left(1-i \Omega h_{1}(z)\right)
$$

Substituting this ansatz into (A.1) we obtain the differential equation for $h_{1}(z)$

$$
h_{1}^{\prime \prime}(z)-\underbrace{\frac{2}{z\left(1-4 \pi^{2} T^{2} z^{2}\right)}}_{p_{1}(z)} h_{1}^{\prime}(z)=-\underbrace{\frac{4 \pi^{2} T^{2}}{\left(1-4 \pi^{2} T^{2} z^{2}\right)}}_{q_{1}(z)}
$$

Lets cast this into a first order differential equation defining $y_{1}(z) \equiv f_{1}^{\prime}(z)$ and consequently $y_{1}^{\prime}(z) \equiv f_{1}^{\prime \prime}(z)$

$$
y_{1}^{\prime}(z)+p_{1}(z) y_{1}(z)=q_{1}(z)
$$

Introducing the integrating factor $I_{1}(z)=\exp \left(\int p_{1}(z) \mathrm{d} z\right)$

$$
\begin{aligned}
y_{1}(z) & =\frac{c_{1}}{I_{1}(z)}+\frac{1}{I_{1}(z)} \int^{z} I_{1}(x) q_{1}(x) \mathrm{d} x \\
& =\underbrace{\frac{c_{1} z^{2}}{1-4 \pi^{2} T^{2} z^{2}}}_{y_{1}^{h}(z)}+\underbrace{\frac{4 \pi^{2} T^{2} z}{1-4 \pi^{2} T^{2} z^{2}}}_{y_{1}^{p}(z)}
\end{aligned}
$$

The homogeneous part of the solution $\left(y_{i}^{h}(z)\right)$ will again be identical in each order in $\Omega$ up to the undetermined coefficient $\left(c_{i}\right)$. This coefficient is fixed by demanding the regularity of $h_{i}$ at the horizon.

$$
\begin{aligned}
h_{1}(z) & =\int y_{1}^{h}(z) \mathrm{d} z+\int y_{1}^{p}(z) \mathrm{d} z \\
& \equiv h_{1}^{h}(z)+h_{1}^{p}(z)
\end{aligned}
$$

Demanding regularity for $h_{1}(z)$ fixes the coefficient of $\log (1-2 \pi T z)$ to zero. One can explicitly calculate the integrals and from that expression sort out the required coefficient. For this case

$$
\begin{aligned}
h_{1}^{h}(z) & =c_{1}\left[-\frac{z}{4 \pi^{2} T^{2}}+\frac{1}{16 \pi^{3} T^{3}}\{\log (1+2 \pi T z)-\log (1-2 \pi T z)\}\right] \\
h_{1}^{p}(z) & =-\frac{1}{2} \log \left(1-4 \pi^{2} T^{2} z^{2}\right) \\
& =-\frac{1}{2}\{\log (1+2 \pi T z)+\log (1-2 \pi T z)\}
\end{aligned}
$$

Clearly setting the coefficient of $\log (1-2 \pi T z)$ to zero we get

$$
c_{1}=-8 \pi^{3} T^{3}
$$

And the solution at this order becomes

$$
h_{1}(z)=\frac{1}{2}[4 \pi T z-2 \log (1+2 \pi T z)]
$$

As has been argued before we can equivalently set the residue of $y_{1}(z)$ at $z=\frac{1}{2 \pi T}$ to zero to fix the value of $c_{1}$. 
Solution up to $\mathcal{O}\left(\Omega^{2}\right)$.

$$
f_{\omega}(z)=\left(1-4 \pi^{2} T^{2} z^{2}\right)^{-i \frac{\Omega}{2}}\left(1-i \Omega h_{1}(z)-\Omega^{2} h_{2}(z)\right)
$$

$h_{1}(z)$ is already known from (A.11). The differential equation for $h_{2}(z)$ reduces to

$$
y_{2}^{\prime}(z)+p_{2}(z) y_{2}(z)=q_{2}(z)
$$

where $p_{2}(z)=p_{1}(z)$ and integrating factor $I_{2}(z)=I_{1}(z)$.

$$
\begin{aligned}
y_{2}(z) & =\frac{c_{2}}{I_{2}(z)}+\frac{1}{I_{2}(z)} \int^{z} I_{2}(x) q_{2}(x) \mathrm{d} x \\
& =\underbrace{\frac{c_{2} z^{2}}{1-4 \pi^{2} T^{2} z^{2}}}_{y_{2}^{h}(z)}+\underbrace{\frac{1}{I_{2}(z)} \int^{z} I_{2}(x) q_{2}(x) \mathrm{d} x}_{y_{2}^{p}(z)}
\end{aligned}
$$

Now making the residue of $y_{2}(z)$ at $z=\frac{1}{2 \pi T}$ to vanish we can fix

$$
c_{2}=8 \pi^{3} T^{3}
$$

The solution at this order

$$
h_{2}(z)=\frac{1}{2}\left[-4 \pi T z+\{\log (1+2 \pi T z)\}^{2}\right]
$$

\section{Solution up to $\mathcal{O}\left(\Omega^{3}\right)$.}

$$
f_{\omega}(z)=\left(1-4 \pi^{2} T^{2} z^{2}\right)^{-i \frac{\Omega}{2}}\left(1-i \Omega h_{1}(z)-\Omega^{2} h_{2}(z)+i \Omega^{3} h_{3}(z)\right)
$$

where $h_{1}, h_{2}$ are known from (A.11) and (A.15). The differential equation for $h_{3}(z)$ or rather $y_{3}(z) \equiv h_{3}^{\prime}(z)$

$$
y_{3}^{\prime}(z)+p_{3}(z) y_{2}(z)=q_{3}(z)
$$

where $p_{3}(z)=p_{1}(z)$ and thus integrating factor $I_{3}(z)=I_{1}(z)$, as before.

$$
\begin{aligned}
y_{3}(z) & =\frac{c_{3}}{I_{3}(z)}+\frac{1}{I_{3}(z)} \int^{z} I_{3}(x) q_{3}(x) \mathrm{d} x \\
& =\underbrace{\frac{c_{3} z^{2}}{1-4 \pi^{2} T^{2} z^{2}}}_{y_{3}^{h}(z)}+\underbrace{\frac{1}{I_{3}(z)} \int^{z} I_{3}(x) q_{3}(x) \mathrm{d} x}_{y_{3}^{p}(z)}
\end{aligned}
$$

Now fixing residue of $y_{3}(z)$ at $z=\frac{1}{2 \pi T}$ to vanish we obtain

$$
c_{3}=0
$$

The functional form of $h_{3}(z)$ is simple unlike the higher dimensional case.

$$
h_{3}(z)=\pi T z\{\log (1+2 \pi T z)\}^{2}-\frac{1}{6}\{\log (1+2 \pi T z)\}^{3}
$$


Therefore in this perturbative expansion the full solution becomes

$$
\begin{aligned}
f_{\omega}^{R}(z)= & \left(1-4 \pi^{2} T^{2} z^{2}\right)^{-i \frac{\Omega}{2}}\left[1-i \Omega\left(\frac{1}{2}(4 \pi T z-2 \log (1+2 \pi T z))\right)\right. \\
& -\Omega^{2}\left(\frac{1}{2}\left(-4 \pi T z+\{\log (1+2 \pi T z)\}^{2}\right)\right) \\
& \left.+i \Omega^{3}\left(\pi T z\{\log (1+2 \pi T z)\}^{2}-\frac{1}{6}\{\log (1+2 \pi T z)\}^{3}\right)\right]
\end{aligned}
$$

Evidently in the zero temperature limit (with very small frequency)

$$
\left.f_{\omega}^{R}(z)\right|_{T \rightarrow 0}=1+\frac{\omega^{2} z^{2}}{2}+i \frac{\omega^{3} z^{3}}{3}
$$

In $r$ co-ordinate

$$
\left.f_{\omega}^{R}(r)\right|_{T \rightarrow 0}=1+\frac{\omega^{2} L^{4}}{2 r^{2}}+i \frac{\omega^{3} L^{6}}{3 r^{3}}
$$

The retarded Green's function at $T=0$ can be calculated using this solution

$$
\begin{aligned}
G_{R}^{0} & \equiv \lim _{r \rightarrow r_{B}} T_{0}(r) f_{-\omega}^{R}(r) \partial_{r} f_{\omega}^{R}(r) \\
& =\lim _{r \rightarrow r_{B}} \frac{1}{2 \pi l_{s}^{2}} \frac{r^{4}}{L^{4}}\left(-\frac{\omega^{2} L^{4}}{r^{3}}-i \frac{\omega^{3} L^{6}}{r^{4}}\right) \\
& =-\frac{\mu \omega^{2}}{2 \pi}-i \frac{\sqrt{\lambda}}{2 \pi} \omega^{3}
\end{aligned}
$$

Thus the renormalized Green's function

$$
G_{R}(\omega) \equiv G_{R}^{0}+\frac{\mu \omega^{2}}{2 \pi}=-i \frac{\sqrt{\lambda}}{2 \pi} \omega^{3}
$$

This matches identically with the leading term in small frequency expansion of (3.8).

Open Access. This article is distributed under the terms of the Creative Commons Attribution License (CC-BY 4.0), which permits any use, distribution and reproduction in any medium, provided the original author(s) and source are credited.

\section{References}

[1] J. de Boer, V.E. Hubeny, M. Rangamani and M. Shigemori, Brownian motion in AdS/CFT, JHEP 07 (2009) 094 [arXiv: 0812.5112] [InSPIRE].

[2] D.T. Son and D. Teaney, Thermal Noise and Stochastic Strings in AdS/CFT, JHEP 07 (2009) 021 [arXiv:0901.2338] [INSPIRE].

[3] P. Banerjee and B. Sathiapalan, Holographic Brownian Motion in $1+1$ Dimensions, Nucl. Phys. B 884 (2014) 74 [arXiv:1308.3352] [InSPIRE].

[4] P. Banerjee, Holographic Brownian Motion at Finite Density, arXiv:1512.05853 [InSPIRE].

[5] E. Caceres, M. Chernicoff, A. Guijosa and J.F. Pedraza, Quantum Fluctuations and the Unruh Effect in Strongly-Coupled Conformal Field Theories, JHEP 06 (2010) 078 [arXiv: 1003.5332] [INSPIRE]. 
[6] Y. Hatta, E. Iancu, A.H. Mueller and D.N. Triantafyllopoulos, Radiation by a heavy quark in $N=4$ SYM at strong coupling, Nucl. Phys. B 850 (2011) 31 [arXiv:1102.0232] [INSPIRE].

[7] M. Chernicoff, A. Guijosa and J.F. Pedraza, The Gluonic Field of a Heavy Quark in Conformal Field Theories at Strong Coupling, JHEP 10 (2011) 041 [arXiv:1106.4059] [INSPIRE].

[8] M. Chernicoff, J.A. Garcia, A. Guijosa and J.F. Pedraza, Holographic Lessons for Quark Dynamics, J. Phys. G 39 (2012) 054002 [arXiv:1111.0872] [INSPIRE].

[9] M. Chernicoff, J.A. Garcia and A. Guijosa, Generalized Lorentz-Dirac Equation for a Strongly-Coupled Gauge Theory, Phys. Rev. Lett. 102 (2009) 241601 [arXiv:0903.2047] [INSPIRE].

[10] M. Chernicoff, J.A. Garcia and A. Guijosa, A Tail of a Quark in N=4 SYM, JHEP 09 (2009) 080 [arXiv:0906.1592] [INSPIRE].

[11] M. Chernicoff and A. Guijosa, Acceleration, Energy Loss and Screening in Strongly-Coupled Gauge Theories, JHEP 06 (2008) 005 [arXiv: 0803.3070] [INSPIRE].

[12] C.P. Herzog, A. Karch, P. Kovtun, C. Kozcaz and L.G. Yaffe, Energy loss of a heavy quark moving through $N=4$ supersymmetric Yang-Mills plasma, JHEP 07 (2006) 013 [hep-th/0605158] [INSPIRE].

[13] W. Fischler, J.F. Pedraza and W. Tangarife Garcia, Holographic Brownian Motion in Magnetic Environments, JHEP 12 (2012) 002 [arXiv:1209.1044] [INSPIRE].

[14] J.M. Maldacena, The large- $N$ limit of superconformal field theories and supergravity, Int. J. Theor. Phys. 38 (1999) 1113 [hep-th/9711200] [INSPIRE].

[15] E. Witten, Anti-de Sitter space and holography, Adv. Theor. Math. Phys. 2 (1998) 253 [hep-th/9802150].

[16] S.S. Gubser, I.R. Klebanov and A.M. Polyakov, Gauge theory correlators from non-critical string theory, Phys. Lett. B 428 (1998) 105 [hep-th/9802109].

[17] E. Witten, Anti-de Sitter space, thermal phase transition and confinement in gauge theories, Adv. Theor. Math. Phys. 2 (1998) 505 [hep-th/9803131] [INSPIRE].

[18] G. Policastro, D.T. Son and A.O. Starinets, The Shear viscosity of strongly coupled $N=4$ supersymmetric Yang-Mills plasma, Phys. Rev. Lett. 87 (2001) 081601 [hep-th/0104066] [INSPIRE].

[19] A. Mikhailov, Nonlinear waves in AdS/CFT correspondence, hep-th/0305196 [INSPIRE].

[20] B.-W. Xiao, On the exact solution of the accelerating string in AdS $S_{5}$ space, Phys. Lett. B 665 (2008) 173 [arXiv:0804.1343] [INSPIRE].

[21] T. Fulton and F. Rohrlich, Classical radiation from a uniformly accelerated charge, Annals Phys. 9 (1960) 499.

[22] D.G. Boulware, Radiation From a Uniformly Accelerated Charge, Annals Phys. 124 (1980) 169 [INSPIRE].

[23] D. Correa, J. Henn, J. Maldacena and A. Sever, An exact formula for the radiation of a moving quark in $\mathcal{N}=4$ super Yang-Mills, JHEP 06 (2012) 048 [arXiv:1202.4455] [INSPIRE]. 
[24] B. Fiol and B. Garolera, Energy Loss of an Infinitely Massive Half-Bogomol'nyi-Prasad-Sommerfeld Particle by Radiation to All Orders in 1/N, Phys. Rev. Lett. 107 (2011) 151601 [arXiv:1106.5418] [INSPIRE].

[25] B. Fiol, B. Garolera and A. Lewkowycz, Exact results for static and radiative fields of a quark in $N=4$ super Yang-Mills, JHEP 05 (2012) 093 [arXiv:1202.5292] [INSPIRE].

[26] B. Fiol, B. Garolera and G. Torrents, Exact momentum fluctuations of an accelerated quark in $N=4$ super Yang-Mills, JHEP 06 (2013) 011 [arXiv:1302.6991] [INSPIRE].

[27] B. Fiol, E. Gerchkovitz and Z. Komargodski, Exact Bremsstrahlung Function in $N=2$ Superconformal Field Theories, Phys. Rev. Lett. 116 (2016) 081601 [arXiv:1510.01332] [INSPIRE].

[28] A. Lewkowycz and J. Maldacena, Exact results for the entanglement entropy and the energy radiated by a quark, JHEP 05 (2014) 025 [arXiv: 1312.5682] [INSPIRE].

[29] A.M. Polyakov, Gauge Fields as Rings of Glue, Nucl. Phys. B 164 (1980) 171 [inSPIRE].

[30] S.W. Hawking and D.N. Page, Thermodynamics of Black Holes in anti-de Sitter Space, Commun. Math. Phys. 87 (1983) 577 [INSPIRE].

[31] D.T. Son and A.O. Starinets, Minkowski space correlators in AdS/CFT correspondence: Recipe and applications, JHEP 09 (2002) 042 [hep-th/0205051] [INSPIRE].

[32] C.P. Herzog and D.T. Son, Schwinger-Keldysh propagators from AdS/CFT correspondence, JHEP 03 (2003) 046 [hep-th/0212072] [INSPIRE].

[33] D.J. Griffiths, Introduction to Electrodynamics, 3rd edition, Prentice Hall (2009). 